\title{
CD30-targeting immunoconjugates and bystander effects
}

Shigeo Masuda, Shigeru Miyagawa, Nagako Sougawa and Yoshiki Sawa

Immunoconjugates, also known as antibody-drug conjugates (ADCs), consist of monocloncal antibodies and cytotoxic drugs (for example, monomethyl auristatin E [MMAE], a potent mitotic spindle formation inhibitor), which are connected by a linker. The elegant Review by Smaglo and colleagues (The development of immunoconjugates for targeted cancer therapy. Nat. Rev. Clin. Oncol. 11, 637-648; 2014) $)^{1}$ explains the details of ADCs, including brentuximab vedotin, an anti-CD30-MMAE immunoconjugate that received FDA approval for the treatment of patients with Hodgkin lymphoma or anaplastic large-cell lymphoma in 2011. Other forms of lymphoma have been intensively investigated to explore whether brentuximab vedotin is an effective treatment for tumours containing cells that present CD30. The high clinical response rates achieved using brentuximab vedotin in patients with lymphoma suggest that this agent is one of the most promising drugs currently available for targeted lymphoma treatment. However, we would like to highlight interesting, and potentially important, findings on the mechanism of action of this targeted therapy, and discuss possible future perspectives.

According to reports, a paradoxical lack of correlation' exists between tumour CD30 expression and clinical response to brentuximab vedotin; ${ }^{2}$ even in tumours in which the percentage of CD30-positive cells is low, the response rate is sometimes high. Although the precise mechanisms underlying this phenomenon remain unknown, some evidence indicates a 'bystander effect', whereby free MMAE is released from dying cells in concentrations sufficient to kill neighbouring tumour cells ${ }^{3}$ either directly or indirectly by altering the tumour microenvironment. This bystander effect might explain the favourable clinical responses associated with brentuximab vedotin. Tumour heterogeneity is increasingly recognized as a key determinant of treatment outcome, and bystander effects of targeted therapy that overcome heterogeneous expression of the target antigen might have important clinical consequences. The bystander effect itself has been demonstrated elsewhere, in a study using BAY 94-9343, a novel ADC targeting mesothelin. ${ }^{4}$ Other ADCs include auristatinbased immunoconjugates targeting tumour necrosis factor receptor superfamily member 17 (also known as B-cell maturation protein; GSK2857916), ${ }^{5} \mathrm{CD} 70$ (SGN-75), ${ }^{6}$ CXC-motif chemokine receptor $4,{ }^{7}$ prostate-specific membrane antigen, ${ }^{8}$ and LIV-1 (also known as ZIP6; SGN-LIV1A), ${ }^{9}$ as well as an anti-CD37-maytansinoid immunoconjugate (IMGN529). ${ }^{10}$ Whether or not these agents have the same bystander effects on surrounding cells would be interesting to know.

CD30 expression is not a predictive marker of clinical response to brentuximab vedotin treatment, therefore, development of a biomarker that can identify patients who respond to this agent is essential. For example, tumours associated with substantial linker digestion-owing to higher lysosomal protease expression-and thus higher local drug concentrations might have the best responses to this agent. Comprehensive analysis should be performed to reveal markers that accurately predict response to treatment, which will inform clinical decision making.

When brentuximab vedotin is used for purging of CD30-expressing cells in vitro during cell processing, repeated refreshing of medium (or washing) in vitro should minimize cytotoxicity owing to bystander effects and could result in reduced cytotoxicity by free MMAE. Such a strategy might be effective for ex vivo tumour purging prior to autologous (non-allogeneic) haematopoietic stem cell transplantation, for example.

Taken together, existing data demonstrate that brentuximab vedotin is a successful treatment for patients with certain types of lymphoma; however, a paradoxical lack of correlation between antigen expression and response might be a result of bystander effects of this treatment, and demands further study.

Department of Cardiovascular Surgery, Osaka University Graduate School of Medicine,

2-2 Yamadaoka, Suita, Osaka 565-0871, Japan (S.M., S.M., N.S., Y.S.).

Correspondence to: Y.S.

sawa-p@surg1.med.osaka u.ac.jp

\section{Ackknowledgements}

The authors express their appreciation to Drs Kaori Okimoto, Satsuki Fukushima and Atsuhiro Saito for their assistance with this correspondence.

Competing interests

The authors declare no competing interests.

1. Smaglo, B. G., Aldeghaither, D. \& Weiner, L. M. The development of immunoconjugates for targeted cancer therapy. Nat. Rev. Clin. Oncol. 11, 637-648 (2014).

2. Horwitz, S. M. et al. Objective responses in relapsed T-cell lymphomas with single-agent brentuximab vedotin. Blood 123, 3095-3100 (2014).

3. Okeley, N. M. et al. Intracellular activation of SGN-35, a potent anti-CD30 antibody-drug conjugate. Clin. Cancer Res. 16, 888-897 (2010).

4. Golfier, S. et al. Anetumab ravtansine: a nove mesothelin-targeting antibody-drug conjugate cures tumors with heterogeneous target expression favored by bystander effect. Mol. Cancer Ther. 13, 1537-1548 (2014).

5. Tai, Y. T. et al. Novel anti-B-cell maturation antigen antibody-drug conjugate (GSK2857916) selectively induces killing of multiple myeloma. Blood 123, 3128-3138 (2014).

6. Tannir, N. M. et al. Novel Phase I doseescalation study of SGN-75 in patients with CD70-positive relapsed/refractory non-Hodgkin lymphoma or metastatic renal cell carcinoma. Invest. New Drugs 32, 1246-1257 (2014).

7. Kularatne, S. A. et al. A CXCR4-targeted sitespecific antibody-drug conjugate. Angew. Chem. Int. Ed. Engl. 53, 11863-11867 (2014).

8. DiPippo, V. A. et al. Efficacy studies of an antibody-drug conjugate PSMA-ADC in patientderived prostate cancer xenografts. Prostate 75, 303-313 (2015).

9. Sussman, D. et al. SGN-LIV1A: a novel antibodydrug conjugate targeting LIV-1 for the treatment of metastatic breast cancer. Mol. Cancer Ther. 13, 2991-3000 (2014).

10. Beckwith, K. A. et al. The CD37-targeted antibody-drug conjugate IMGN529 is highly active against human CLL and in a novel CD37 transgenic murine leukemia model. Leukemia 28, 1501-1510 (2014). 